USM-TH-193

\title{
Extended Black Box Theorem for Lepton Number and Flavor Violating processes
}

\author{
Martin Hirsch, ${ }^{1}$ Sergey Kovalenko ${ }^{2}$ and Ivan Schmidt ${ }^{2}$ \\ 1 AHEP Group, Institut de Física Corpuscular \\ C.S.I.C./Universitat de València \\ Edificio Institutos de Paterna, Apt 22085, E-46071 Valencia, Spain \\ ${ }^{2}$ Departamento de Física, Universidad Técnica Federico Santa María, \\ Casilla 110-V, Valparaíso, Chile
}

\begin{abstract}
We revisit the well known "Black Box" theorem establishing a fundamental relation between the amplitude of neutrinoless double beta decay and the effective Majorana neutrino mass. We extend this theorem to the general case of arbitrary lepton number and lepton flavor violating (LFNV) processes and to the three generation Majorana neutrino mass matrix. We demonstrate the existence of a general set of one-to-one correspondence relations between the effective operators generating these processes, and elements of the neutrino mass matrix, such that if one of these two quantities vanishes the other is guaranteed to vanish as well, and moreover, if one of these quantities is non-zero the other is guaranteed to be non-zero. We stress that this statement remains valid even in the presence of arbitrary new physics contributions. As a particularly important example, we then show that neutrino oscillation data imply that neutrinoless double beta decay must occur at a certain non-zero rate.
\end{abstract}

PACS: 13.35.Dx,13.35.Hb,14.60.Pq,14.60.St

KEYWORDS: new physics, lepton flavor violation, neutrino. 


\section{Introduction}

In the past few years neutrino oscillation experiments [1] have, for the first time, provided unambiguous evidence for lepton flavor violation (LFV). The current status of the experimental data can be briefly summarized as follows [2]. Two neutrino mass squared differences and two neutrino angles are known to be nonzero. These are the atmospheric neutrino mass, $\Delta m_{\text {Atm }}^{2}=(2.0-3.2)\left[10^{-3}\right.$ $\mathrm{eV}^{2}$, and angle, $\sin ^{2} \theta_{\mathrm{Atm}}=(0.34-0.68)$, as well as the solar neutrino mass $\Delta m_{\odot}^{2}=(7.1-8.9)\left[10^{-5} \mathrm{eV}^{2}\right]$, and angle, $\sin ^{2} \theta_{\odot}=(0.24-0.40)$, all numbers at $3 \sigma$ c.l. For the remaining neutrino angle, often called the reactor neutrino angle $\theta_{R}$, and for the overall neutrino mass scale only upper limits exist $[2,3]$.

Interestingly, the observed LFV can not exist isolated only in the neutrino sector. It inevitably is carried over to the sector of charged leptons via the LFV charged current loop with virtual neutrinos and should manifest itself in the form of LFV processes with charged leptons, such as $\mu, \tau$ and meson LFV decays, $\mu^{-}-e^{-}$nuclear conversion, etc. Thus, given the observation of neutrino oscillations, the existence of yet unobserved LFV processes with charged leptons is expected.

However, the LFV in the charged lepton sector may also receive direct contributions from non-SM interactions of new physics. These may cancel the contribution of the loop induced effect related to neutrino oscillations. Such a cancellation is phenomenologically unnatural, but possible if there exists some symmetry protecting this cancellation. This observation gives rise to the question as to whether the observation of neutrino oscillations guarantees non-zero rates of some LFV processes with charged leptons and vice versa. In view of the experimental observation of neutrino oscillations the latter part of this question may be considered a purely theoretical curiosity but it may reveal some generic relations between the neutrino mass matrix and LFV processes with charged leptons.

One particular example of such kind of relation has been known for some time. It is generally referred to as the "Black Box" theorem [4], and relates the effective Majorana neutrino mass and the amplitude of neutrinoless double beta decay. In essence, it establishes a one-to-one correspondence between these two quantities, such that if one of them vanishes the other is guaranteed to vanish and if one of these quantities is non-zero the other must be non-zero as well. ${ }^{1}$ The crucial point of the "Black Box" theorem is that it remains valid in the presence of arbitrary contributions of new physics. Note, however, that the existing version

\footnotetext{
${ }^{1}$ A supersymmetric version of the "Black Box" theorem has been formulated in Ref. [5], extending this relation also to the lepton number violating scalar neutrino mass.
} 
of the "Black box" theorem [4] is limited to the relation between neutrinoless double beta decay and the Majorana property of the $\nu_{e}$ neutrino flavor, without properly taking into account its mixing with (at least two) other neutrino flavor states $\nu_{\mu}$ and $\nu_{\tau}$.

In the present paper we therefore revisit the "Black Box" theorem and extend it to the general case of arbitrary lepton number and flavor violating (LFNV) processes and the three-generation Majorana neutrino mass matrix. Applying symmetry arguments we will show that there exists a one-to-one correspondence between the amplitudes of the LFNV processes and the corresponding entries of the Majorana neutrino mass matrix. The same symmetry arguments allow us to formulate self-consistency conditions for the Majorana neutrino mass matrix, thus restricting its allowed index structure. We study in more details the specific case of neutrinoless double beta decay and the corresponding $M_{e e}^{\nu}$ element of the Majorana neutrino mass matrix. We show that the self-consistency condition, in combination with neutrino oscillation data, exclude the case $M_{e e}^{\nu}=0$, guaranteeing that neutrinoless double beta decay must occur at a non-zero rate.

\section{LFNV Processes and Neutrino mass Matrix}

Here we are going to prove a generic set of one-to-one correspondence relations between LFV processes with total lepton number violation (LFNV) $\Delta L=\Delta\left(L_{\alpha}+\right.$ $\left.L_{\beta}\right)=2$ and the corresponding entries of the Majorana neutrino mass matrix. We consider baryon number conserving processes in which LFNV manifests itself via two external charged leptons. In addition, these processes may contain any set of external SM particles satisfying certain conditions to be specified below. For convenience we put all these additional particles to the initial states of the LFNV processes while the two charged leptons appear in the final states. Nevertheless our conclusions are valid for any $\Delta L=2$ process, as will be seen later on.

Without loss of generality we focus on $\Delta L=2$ processes of the form (see Fig 1(a).): $\Phi_{k} \rightarrow l_{\alpha} l_{\beta}$, where $\Phi_{k}$ denotes certain color-singlet subsets of external particles with $B=0, L_{\alpha}=0$ and total electric charge $Q=-2$. The difference between the processes with the same lepton flavor structure, $l_{\alpha} l_{\beta}$, is due to the difference of their external particle subsets $\Phi_{k}$ marked with different subscript $k$. The possible subsets considered are of the following two types:

$$
\Phi_{0}=W^{-} W^{-}, \quad \Phi_{k}=(\bar{u} d)(\bar{u} d)\{(\bar{u} u),(\bar{d} d), \ldots\}\left\{\left(\bar{l}_{\alpha} l_{\alpha}\right), \gamma, Z\right\} .
$$

The second type of sets must contain two pairs of anti-up and down quarks of 
arbitrary generation in order to provide $Q=-2$. They may or may not be accompanied by an arbitrary neutral set of particles.

We write down the effective Lagrangian describing the LFNV processes in the following schematic form:

$$
\mathcal{L}^{\Delta L=2}=\Phi_{k} \bar{l}_{\alpha} \Gamma_{\alpha \beta}^{(k)} l_{\beta}^{c}+g \bar{l}_{\alpha} \gamma^{\mu} P_{L} \nu_{\alpha} W_{\mu}^{-}+\Phi_{k} W_{\mu}^{-} S_{k}^{\mu \nu} W_{\nu}^{-}+\mathcal{L}^{\prime}+\text { h.c. }
$$

Here, $P_{L}=\left(1-\gamma_{5}\right) / 2$. The first effective operator generates amplitudes for $\Phi_{k} \rightarrow l_{\alpha} l_{\beta}$ processes and may receive contributions from SM interactions with Majorana neutrino mass insertions (to be discussed in the next section) and possibly from some physics beyond the SM. The vertex structure $\Gamma^{(k)}$ depends on the concrete realization of this operator. The second term is the usual SM leptonic charged current interaction. The third term represents the SM interactions of the sets of fields $\Phi_{k}$ with W-bosons. This term plays an important role in our analysis and is always induced by the SM interactions. This is guaranteed by the structure of the field set $\Phi_{k}$ given in Eq. (1) and directly follows from the presence of the two $\bar{u} d$ pairs which have SM couplings to $W^{-}$. The term $\mathcal{L}^{\prime}$ denotes any interactions beyond the SM, whose explicit form is irrelevant for our analysis. The particular realization of the above terms depends on the field set $\Phi_{k}$ and determines the vertex structure $S_{k}$. The explicit form of $\Gamma^{(k)}$ and $S_{k}$ are also irrelevant for our subsequent reasoning. In what follows we denote the first and second effective operators in Eq. (2) by $\hat{\Gamma}_{\alpha \beta}^{(k)}$ and $\hat{S}_{k}$, respectively.

Majorana mass terms for the three left handed neutrinos $\nu_{\alpha}=\nu_{e}, \nu_{\mu}, \nu_{\tau}$ in 4-component notations can be written as

$$
\mathcal{L}_{\alpha \beta}^{M}=-\frac{1}{2} \overline{\nu_{\alpha}^{c}} M_{\alpha \beta}^{\nu} P_{L} \nu_{\beta}+\text { h.c. }
$$

It is obvious that neutrinos contribute to the operator $\hat{\Gamma}_{\alpha \beta}^{(k)}$ in Eq. (2) via the well known diagram in Fig. 1(a), leading to the following expression for the vertex function

$$
\begin{aligned}
\Gamma_{\alpha \beta}^{(k)}\left(x_{1}-x_{2}, \ldots\right) & \sim \int \frac{d^{4} q}{(2 \pi)^{4}} M_{\alpha m}^{\nu}\left(q^{2}-M^{\nu \dagger} M^{\nu}\right)_{m \beta}^{-1} e^{-i q\left(x_{1}-x_{2}\right)}= \\
& =\int \frac{d^{4} q}{(2 \pi)^{4}} q^{-2} M_{\alpha m}^{\nu}\left(\delta_{m \beta}+\sum_{n=1}^{\infty} q^{-2 n}\left(M^{\nu \dagger} M^{\nu}\right)_{m \beta}^{n}\right) e^{-i q\left(x_{1}-x_{2}\right)} .
\end{aligned}
$$

Here we have formally expanded the denominator in the ratio $M_{(\nu)}^{2} / q^{2}$. The terms of this expansion correspond, in the flavor basis, to the contributions of all possible mass insertions in the intermediate neutrino line, see Fig.1(a). The number of 
insertions must be odd in order to have total lepton number violation $\Delta L=2$, since each Majorana mass insertion introduces $\Delta L= \pm 2$. Thus, the leading order neutrino contribution to the operator $\hat{\Gamma}_{\alpha \beta}^{(k)}$ is proportional to the $M_{\alpha \beta}^{\nu}$ entry of the Majorana neutrino mass matrix. However, this operator receives higher order contributions from the other entries which can generate it even in the absence of an $M_{\alpha \beta}^{\nu}$ entry. The infinite serie in Eq. (4) can be represented in a compact form on the basis of the Hamilton-Kelly theorem [7], which asserts that any analytic function of an $n \times n$ matrix is equivalent to an n- 1 order polynomial of this matrix. Applying this theorem to Eq. (4) we get

$$
\Gamma_{\alpha \beta}^{(k)}=c_{0} M_{\alpha \beta}^{\nu}+c_{1} M_{\alpha k}^{\nu}\left(M^{\nu \dagger} M^{\nu}\right)_{k \beta}+c_{2} M_{\alpha k}^{\nu}\left(M^{\nu \dagger} M^{\nu}\right)_{k \beta}^{2}+\Gamma^{\prime} .
$$

The coefficients $c_{n}$ depend on the mass eigenvalues $m_{\nu_{1,2,3}}^{2}$ of the matrix $M^{\nu \dagger} M^{\nu}$. Here, in addition to the first three terms following from Eq. (4) we allowed for a term $\Gamma^{\prime}$ representing any possible contributions other than those of Fig. 1(a). The above formula represents the net result of all possible mass insertions. The advantage of the representation (5) is that it specifies all the irreducible sets of non-trivial LFV transitions, into which any other LFV transition in the infinite sum of Eq. (4) can be decomposed. We will use this fact later.

Now consider the contribution of the operator $\hat{\Gamma}_{\alpha \beta}^{(k)}$ in Eq. (2) to the Majorana neutrino mass matrix $M_{\alpha \beta}^{\nu}$ in Eq. (3). This contribution is generated by the 1PI self-energy diagrams in Fig. 1(b) with two SM charged current vertices. In leading order, taking into account all non-trivial lepton flavor transitions, we write the corresponding contribution to the Majorana neutrino mass matrix in a form similar to that of Eq. (5):

$$
M_{\alpha \beta}^{\nu}=\Sigma_{0}\left\langle\Gamma_{\alpha \beta}\right\rangle+\Sigma_{1}\left\langle\Gamma_{\alpha m}\left(\Gamma^{\dagger} \Gamma\right)_{m \beta}\right\rangle+\Sigma_{2}\left\langle\Gamma_{\alpha m}\left(\Gamma^{\dagger} \Gamma\right)_{m \beta}^{2}\right\rangle+M^{\prime}
$$

where in the brackets \langle\rangle a convolution in coordinate space and matrix multiplication in flavor space are implied. As in Eq. (5), here we also allowed for a term $M^{\prime}$ representing any possible contributions other than in Fig. 1(b). The explicit form of the terms in Eqs. (6),(5) is not important for our subsequent analysis. Decisive, however, is the observation that the operator $\hat{\Gamma}_{\alpha \beta}^{(k)}$ from Eq. (2) and the Majorana neutrino mass matrix $M_{\alpha \beta}^{\nu}$ defined in Eq. (3) mutually contribute to each other. 


\section{Extended "Black Box Theorem"}

Now we are in a position to prove the Extended "Black Box" Theorem establishing a one-to-one correspondence between the amplitudes of LNFV processes and the entries of the Majorana neutrino mass matrix. First, let us explore the consequences of the assumption $M_{\alpha \beta}^{\nu}=0$ for $\hat{\Gamma}_{\alpha \beta}^{(k)}$ and vice versa $\hat{\Gamma}_{\alpha \beta}^{(k)}=0$ for $M_{\alpha \beta}^{\nu}$. To this end let us consider Eq. (6) for the former case and Eq. (5) for the latter one. The condition $M_{\alpha \beta}^{\nu}=0$ in Eq. (6) implies that the sum of the terms on the r.h.s. must vanish. This can happen either if each of the four terms vanish individually or if these four non-zero terms cancel each other. The latter case of an accidental self-cancellation is unnatural, since it is in general unstable with respect to radiative corrections. The same arguments can be applied to Eq. (5). Thus, on the basis of naturalness arguments alone one may expect from Eqs. (5) and (6) that $M_{\alpha \beta}^{\nu}=0$ requires $\hat{\Gamma}_{\alpha \beta}^{(k)}=0$ and vice versa. This conclusion may be circumvented only if there exists a symmetry protecting the cancellation to all orders of perturbation theory.

Let us examine if any global symmetry of this type in a theory with the Lagrangian Eq. (2), which includes both SM and beyond the SM interactions, can exist. Such a symmetry should be associated with a unitary transformation of the fields realizing representations of a global group, which we denote by $G_{\eta}$. Suppose under this symmetry the fields transform as

$$
W^{-} \stackrel{G_{\eta}}{\longrightarrow} \eta_{W} \cdot W^{-}, \quad l_{\alpha} \stackrel{G_{\eta}}{\longrightarrow} \eta_{\alpha}^{l} \cdot l_{\alpha}, \quad \nu_{\alpha} \stackrel{G_{\eta}}{\longrightarrow} \eta_{\alpha}^{\nu} \cdot \nu_{\alpha}, \quad \Phi_{k} \stackrel{G_{\eta}}{\longrightarrow} \eta_{k} \cdot \Phi_{k},
$$

with $\eta_{a} \eta_{a}^{\dagger}=1$. Terms in Eq. (2) will then be allowed (forbidden) by the symmetry if the corresponding product of $\eta$ factors is equal to 1 (different from 1 ). The group $G_{\eta}$ is not completely arbitrary since it must at least be consistent with the SM part of the Lagrangian (2). The invariance of the second and third terms in Eq. (2) with respect to $G_{\eta}$ requires:

$$
\eta_{W}^{2} \eta_{k}=1, \quad \eta_{W} \eta_{\alpha}^{l \dagger} \eta_{\alpha}^{\nu}=1
$$

The effective operators $\hat{\Gamma}_{\alpha \beta}^{(k)}$ generating the processes $\Phi_{k} \rightarrow l_{\alpha} l_{\beta}$ and the Majorana neutrino mass terms transform under $G_{\eta}$ as

$$
\begin{aligned}
& \hat{\Gamma}_{\alpha \beta}^{(k)} \stackrel{G_{\eta}}{\longrightarrow} \eta_{\alpha \beta}^{k} \cdot \hat{\Gamma}_{\alpha \beta}^{(k)}, \quad \text { with } \eta_{\alpha \beta}^{k}=\eta_{\alpha}^{l} \cdot \eta_{\beta}^{l} \cdot \eta_{k}, \\
& \mathcal{L}_{\alpha \beta}^{M} \stackrel{G_{\eta}}{\longrightarrow} \eta_{\alpha \beta}^{\nu} \cdot \mathcal{L}_{\alpha \beta}^{M}, \quad \text { with } \eta_{\alpha \beta}^{\nu}=\eta_{\alpha}^{\nu} \cdot \eta_{\beta}^{\nu} .
\end{aligned}
$$

From condition (8) it follows that $\eta_{k}=\left(\eta_{W}^{\dagger}\right)^{2}$ and $\eta_{\alpha}^{l}=\eta_{\alpha}^{\nu} \eta_{W}$ and thus

$$
\eta_{\alpha \beta}^{k}=\eta_{\alpha \beta}^{\nu} \quad \text { for } \quad \forall k \text {. }
$$


We repeat that terms in the Lagrangian Eqs. (2) and (3) are allowed by $G_{\eta}$ if the corresponding transformation factor of this term is $\eta=1$ and forbidden if $\eta \neq 1 .^{2}$ Eq. (11) proves a general one-to-one correspondence between the effective operators $\hat{\Gamma}_{\alpha \beta}^{(k)}$, generating the processes $\Phi_{k} \rightarrow l_{\alpha} l_{\beta}$, and the elements of the neutrino mass matrix $M_{\alpha \beta}^{\nu}$. According to Eq. (11), the effective operator $\hat{\Gamma}_{\alpha \beta}^{(k)}$ and the corresponding element of the mass matrix $M_{\alpha \beta}^{\nu}$ are related such that if one of these two quantities vanishes the other is guaranteed to vanish, and vice versa if one of these quantities is non-zero the other one is non-zero as well. The crucial point of this statement is that it is valid in the presence of any contributions of new physics represented in Eqs. (2), (5) and (6) by $\mathcal{L}^{\prime}, \Gamma^{\prime}$ and $M^{\prime}$. Schematically one can express these relations as:

$$
\left(\Phi_{k} \nrightarrow l_{\alpha} l_{\beta}\right) \hat{\Gamma}_{\alpha \beta}^{(k)}=0 \leftrightarrow M_{\alpha \beta}^{\nu}=0, \quad\left(\Phi_{k} \rightarrow l_{\alpha} l_{\beta}\right) \hat{\Gamma}_{\alpha \beta}^{(k)} \neq 0 \leftrightarrow M_{\alpha \beta}^{\nu} \neq 0,
$$

This result is valid not only for the processes of type $\Phi_{k} \rightarrow l_{\alpha} l_{\beta}$ but for any $\Delta L=\Delta\left(L_{\alpha}+L_{\beta}\right)=2$ process like $\Phi_{k}^{(1)} \rightarrow \Phi_{n}^{(2)} l_{\alpha} l_{\beta}, \Phi_{k}^{(1)} \bar{l}_{\alpha} \rightarrow \Phi_{n}^{(2)} l_{\beta}$, because our conclusions are based on the analysis of the effective operator $\hat{\Gamma}_{\alpha \beta}^{(k)}$, which generates all these processes.

Eq. (12) represents a direct extension of the flavor blind "Black Box" Theorem [4] to the case involving arbitrary LNFV. However, due to the possibility of multiple LNFV transitions, in the latter case there are additional indirect relations between $\Phi_{k} \rightarrow l_{\alpha} l_{\beta}$ and $M_{k n}^{\nu}$ for $k \neq \alpha, n \neq \beta$. Let us turn again to Eqs. (5) and (6). The relations Eq. (12) correspond to the leading order terms on the r.h.s. of Eqs. (5) and (6) when $\hat{\Gamma}_{\alpha \beta}^{(k)}$ and $M_{\alpha \beta}^{\nu}$ are directly proportional to each other. However, according to Eq. (5) and (6), the second and third (next-toleading) terms introduce new relations between $\hat{\Gamma}_{\alpha \beta}^{(k)}$ and $M_{m n}^{\nu}$ for $m \neq \alpha, n \neq \beta$. One can find all these residual relations using the above symmetry arguments. First, using Eqs. (9)-(11), the following relations for the $G_{\eta}$ symmetry factors are obtained

$$
\begin{array}{ll}
\eta_{\alpha \beta}^{\nu}=\eta_{\alpha n}^{k} \eta_{m n}^{k \dagger} \eta_{m \beta}^{k}, & \eta_{\alpha \beta}^{k}=\eta_{\alpha n}^{\nu} \eta_{m n}^{\nu \dagger} \eta_{m \beta}^{\nu}, \\
\eta_{\alpha \beta}^{\nu}=\eta_{\alpha n}^{k} \eta_{m n}^{k \dagger} \eta_{m l}^{k} \eta_{p l}^{k}{ }^{\dagger} \eta_{p \beta}^{k}, & \eta_{\alpha \beta}^{k}=\eta_{\alpha n}^{\nu} \eta_{m n}^{\nu \dagger} \eta_{m l}^{\nu} \eta_{p l}^{\nu \dagger} \eta_{p \beta}^{\nu},
\end{array}
$$

These relations follow from the fact that $\eta_{m n}^{k}=\eta_{m n}^{\nu}=\eta_{m}^{\nu} \eta_{n}^{\nu}$ and $\eta_{i}^{\nu} \eta_{i}^{\nu \dagger}=1$. In principle, one can write down an infinite set of such relations involving multiple

\footnotetext{
${ }^{2}$ Similar arguments have been used previously to prove a one-to-one correspondence between the amplitude of neutrinoless double beta decay, Majorana neutrino [4] and sneutrino [5] masses as well as between different LFNV processes [6].
} 
LNFV transitions corresponding to the mass insertions in Fig. 1(a), given by the infinite series in Eq. (4) as well as the self-energy insertions to the massless neutrino propagator of Fig. 1(b). However, as we already have noted the irreducible set of transitions is given by the first three terms of Eqs. (5) and (6), as follows from the Hamilton-Kelly theorem. Eqs. (11), (13) and (14) represent a complete set of relations between the transformation $\eta$-factors corresponding to these terms. In fact, let us consider Eq. (5) and observe that the field transformations in Eqs. (7) are equivalent to the transformations of the vertex function and neutrino mass matrix: $\Gamma_{\alpha \beta}^{(k)} \stackrel{G_{\eta}}{\longrightarrow} \eta_{\alpha \beta}^{k} \cdot \Gamma_{\alpha \beta}^{(k)}, M_{\alpha \beta}^{\nu} \stackrel{G_{\eta}}{\longrightarrow} \eta_{\alpha \beta}^{\nu} \cdot M_{\alpha \beta}^{\nu}$. Both sides of Eq. (5) must equally transform under $G_{\eta}$. The necessary and sufficient conditions to satisfy this requirement are given by Eqs. (11), (13) and (14). In other words they represent a complete set of non-trivial relations between the symmetry $\eta$-factors. Another way to demonstrate the completeness of the relations Eqs. (11), (13) and (14) is given by combinatorial analysis. In the three generation case under consideration it is easy to show that any relationships other than the type given in Eqs. (13)-(14) can be decomposed into blocks corresponding to irreducible sets of $\eta$-factors on the r.h.s. of these equations.

Thus it is sufficient to consider Eq. (13)-(14), together with Eq. (11), in order to find all possible relations between $\hat{\Gamma}^{(k)}$ and $M^{\nu}$, following from our symmetry arguments. The information provided by Eqs. (13)-(14) can be expressed in various ways. We formulate the consequences of these equations in the form of the following question: what are the minimal sets of the LFNV processes to be observed experimentally that are sufficient to prove that $M_{\alpha \beta}^{\nu} \neq 0$ ? Naturally, the observation of $\Phi_{k} \rightarrow l_{\alpha} l_{\beta}$ would establish this fact as evidenced by Eqs. (12). However, there exist other sets of processes $\Phi_{k} \rightarrow l_{m} l_{n}$ with $m \neq \alpha$ and/or $n \neq \beta$, whose observation would also establish $M_{\alpha \beta}^{\nu} \neq 0$. The complete lists of such processes corresponding to each entry of $M_{\alpha \beta}^{\nu}$ can be derived from Eqs. (13)-(14). Here for illustration we only show the complete list of LFNV processes necessary to establish that $M_{e e}^{\nu} \neq 0$. In this case there are five independent sets of experiments shown in the curl brackets:

$$
M_{e e}^{\nu} \neq 0 \longleftarrow\left\{\begin{array}{l}
\{(e \mu),(\mu \mu)\},\{(e \tau),(\tau \tau)\},\{(e \mu),(\mu \tau),(e \tau)\} \\
\{(e \mu),(\mu \tau),(\tau \tau)\},\{(e \tau),(\mu \tau),(\mu \mu)\} .
\end{array}\right.
$$

Here we denoted the process $\Phi_{k} \rightarrow l_{m} l_{n}$ by $(m n)$, where $m \neq e$ and/or $n \neq e$. The first three sets of experiments follow from Eq. (13) and the last two from Eq. (14). For instance, the observation of both $\Phi_{k} \rightarrow l_{e} l_{\mu}$ and $\Phi_{k} \rightarrow l_{\mu} l_{\mu}$ would establish $M_{e e}^{\nu} \neq 0$, etc. In practice, information of this type might become useful 
in a situation when for some (experimental) reasons the observation of certain $\Phi_{k} \rightarrow l_{\alpha} l_{\beta}$ processes is not possible. In the above example this is $\Phi_{k} \rightarrow l_{e} l_{e}$.

Formally the Extended Black Box Theorem is formulated as Eqs. (11) and (13)-(14), allowing one to extract various relations of the above discussed type between LFNV processes and entries of the Majorana neutrino mass matrix $M^{\nu}$.

\section{Neutrinoless double beta decay}

We will now apply the symmetry arguments of the previous section to analyze the structure of the Majorana neutrino mass matrix itself. As the phenomenologically most relevant lepton number violating process, we will concentrate on the consequences for neutrinoless double beta decay $(0 \nu \beta \beta)$. As is well-known, Majorana neutrino masses induce a $0 \nu \beta \beta$ decay amplitude proportional to

$$
\begin{aligned}
\left\langle m_{\nu}\right\rangle & =\sum_{j} U_{e j}^{2} m_{j} \\
& \equiv M_{e e}^{\nu}
\end{aligned}
$$

Here, the sum includes all light neutrino mass eigenstates. The second relation, i.e. that $0 \nu \beta \beta$ decay is sensitive to the $\left(\nu_{e}-\nu_{e}\right)$ element of the neutrino mass matrix in flavor space, follows from a straightforward calculation.

From the discussion presented above it is clear that $M_{e e}^{\nu} \equiv 0$ requires $\eta_{e e}^{\nu} \neq 1$. We will now show that $\eta_{e e}^{\nu} \neq 1$ is inconsistent with data from neutrino oscillation experiments. The argument is essentially a proof by contradiction: construct all possible neutrino mass matrices consistent with $\eta_{e e}^{\nu} \neq 1$; if none of the resulting matrices can explain all oscillation data, $M_{e e}^{\nu}$ must be non-zero.

As an example, assume $\eta_{e e}^{\nu}=\eta_{e}^{\nu} \eta_{e}^{\nu} \neq 1$. Choose $\eta_{e \mu}^{\nu}=\eta_{e}^{\nu} \eta_{\mu}^{\nu}=1$ and $\eta_{e \tau}^{\nu}=$ $\eta_{e}^{\nu} \eta_{\tau}^{\nu}=1$. It follows that $\eta_{\mu}^{\nu}=\left(\eta_{e}^{\nu}\right)^{*}$ and $\eta_{\tau}^{\nu}=\left(\eta_{e}^{\nu}\right)^{*}$, as well as $\eta_{\mu \mu}^{\nu} \neq 1, \eta_{\mu \tau}^{\nu} \neq 1$ and $\eta_{\tau \tau}^{\nu} \neq 1$. Thus the resulting mass matrix has the structure ${ }^{3}$

$$
\mathcal{M}_{\nu}^{(1)}=\left(\begin{array}{lll}
0 & x & y \\
x & 0 & 0 \\
y & 0 & 0
\end{array}\right) .
$$

In a completely analogous way one can find all possible neutrino mass matrices consistent with $M_{e e}^{\nu}=0$ and with a maximal number of non-zero entries. In

\footnotetext{
${ }^{3}$ We stress that the zeros in these matrices are exact, since we assume they are enforced by a symmetry.
} 
addition to $\mathcal{M}_{\nu}^{(1)}$, the other possibilities have the following structures

$$
\begin{array}{ll}
\mathcal{M}_{\nu}^{(2)}=\left(\begin{array}{lll}
0 & 0 & 0 \\
0 & x & y \\
0 & y & z
\end{array}\right) & \mathcal{M}_{\nu}^{(3)}=\left(\begin{array}{lll}
0 & x & 0 \\
x & 0 & y \\
0 & y & 0
\end{array}\right) \\
\mathcal{M}_{\nu}^{(4)}=\left(\begin{array}{lll}
0 & 0 & y \\
0 & x & 0 \\
y & 0 & 0
\end{array}\right) & \mathcal{M}_{\nu}^{(5)}=\left(\begin{array}{lll}
0 & x & 0 \\
x & 0 & 0 \\
0 & 0 & y
\end{array}\right)
\end{array}
$$

As promised, none of these five matrices is consistent with the data. $\mathcal{M}_{\nu}^{(1)}$ and $\mathcal{M}_{\nu}^{(3)}$ have eigenvalues such that one $\Delta m^{2} \equiv 0 . \mathcal{M}_{\nu}^{(2)}$ leads to a solar angle $\theta_{\odot} \equiv 0$ and $\mathcal{M}_{\nu}^{(5)}$ predicts the atmospheric angle $\theta_{\text {Atm }} \equiv 0$. And, finally, $\mathcal{M}_{\nu}^{(4)}$ predicts the reactor angle to be maximal and $\theta_{\odot} \equiv 0 \equiv \theta_{\text {Atm }}$.

As we have already shown, $0 \nu \beta \beta$ decay must occur at certain non-zero rate if $M_{e e}^{\nu} \neq 0$ and is forbidden if $M_{e e}^{\nu} \equiv 0$. We can conclude that, with the assumption that neutrinos indeed are Majorana particles, current neutrino oscillation data show that neutrinoless double beta decay must occur with a non-zero rate.

\section{Conclusions}

We have proven a generic set of one-to-one correspondence relations between LFV processes with total lepton number violation (LFNV) $\Delta L=\Delta\left(L_{\alpha}+L_{\beta}\right)=2$ and the corresponding entries of the Majorana neutrino mass matrix. These relations are an extension of the well known "Black Box" theorem, establishing a fundamental relation between the amplitude of neutrinoless double beta decay and the effective Majorana neutrino mass, to the general case of arbitrary lepton number and lepton flavor violating (LFNV) processes and the three generation Majorana neutrino mass matrix. As a particularly interesting application of this theorem we have shown that neutrino oscillation data imply that neutrinoless double beta decay must occur at a certain non-zero rate.

\section{Acknowledgments}

This work was supported by the FONDECYT projects 1030244 and 1030355, by Spanish grant FPA2005-01269 and by the European Commission Human Po-

tential Program RTN network MRTN-CT-2004-503369, as well as the EU Net- 
work of Astroparticle Physics (ENTApP) WP1. M.H. is supported by an MCyT Ramon y Cajal contract.

\section{References}

[1] Y. Fukuda et al. [Super-Kamiokande Collaboration], Phys. Rev. Lett. 81, 1562 (1998) [arXiv:hep-ex/9807003]; Q. R. Ahmad et al. [SNO Collaboration], Phys. Rev. Lett. 89, 011301 (2002) [arXiv:nucl-ex/0204008]; K. Eguchi et al. [KamLAND Collaboration], Phys. Rev. Lett. 90, 021802 (2003) [arXiv:hep-ex/0212021].

[2] M. Maltoni, T. Schwetz, M. A. Tortola and J. W. F. Valle, New J. Phys. 6 (2004) 122 [arXiv:hep-ph/0405172]. (V5) in the archive provides updated numbers taking into account all relevant data as of June 2006.

[3] S. Eidelman et al. [Particle Data Group], Phys. Lett. B 592, 1 (2004).

[4] J. Schechter and J.W.F. Valle, Phys.Rev. D 25, 2951 (1982); J.F. Nieves, Phys.Lett. B 147, 375 (1984); E. Takasugi, Phys.Lett. B 149, 372 (1984); B. Kayser, in Proc. of the XXIII Int. Conf on High Energy Physics, ed. S. Loken (World Scientific Singapore, 1987), p. 945; S.P. Rosen, UTAPHY-HEP-4 and hep-ph/9210202.

[5] M. Hirsch, H.V. Klapdor-Kleingrothaus and S.G. Kovalenko, Phys. Lett. B 398, 311 (1997); Phys.Rev. D 57, 1947 (1998).

[6] S. Kovalenko, I. Schmidt, arXiv:hep-ph/0512086.

[7] G.A. Korn and Th.M. Korn, "Mathematical Handbook for Scientists and Engineers", McGraw-Hill, New York San Fransisco Toronto London Sidney, 1968. 


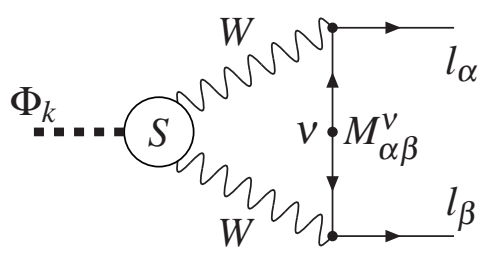

(a)

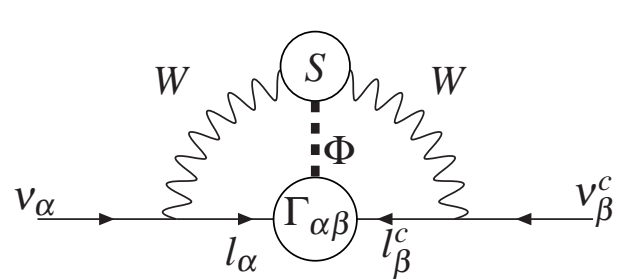

(b)

Figure 1: (a) Contribution of the $M_{\alpha \beta}^{\nu}$ entry of the Majorana neutrino mass matrix to the effective LFNV vertex $\Gamma_{\alpha \beta}$ and visa versa (b) contribution of $\Gamma_{\alpha \beta}$ to $M_{\alpha \beta}^{\nu}$. 